\title{
Using a parametric 3D assembly of a GTE combustion chamber to quickly generate its computed sector
}

\author{
Maksim Nekhoroshev ${ }^{1}$, Mikhail Orlov ${ }^{1}$, and Aleksandr Ryazanov ${ }^{1, *}$ \\ ${ }^{1}$ Samara University, 34 Moskovskoye Shosse, Samara, 443086, Russia
}

\begin{abstract}
The paper describes the development of a parametric 3D CAD assembly of a small-size GTD combustion chamber. Based on the chamber model, we derive the internal CAD volume of the designed single-burner sector. The created parametric models are auto-adjustable by an external excel spreadsheet controlling their parameters. The 3D combusion chamber assembly and the single-burner sector can further be used for optimizing the geometry of flame tubes and nozzles, arranging the sustainable fuel burning space, for the spatial distribution of air flows, and for other design tasks.
\end{abstract}

\section{Introduction}

Combusion chambers (CC) of small-size gas-turbine engines (SGTE) have to meet a number of seemingly mutually exclusive requirements such as: the specified level of circumferential and radial non-uniformity of output temperature fields, a wide range of operating parameters without flame failure, low total pressure losses, a high combustion efficiency, minimum emission of unburned hydrocarbons, etc. This is why designing and finishing combusion chambers is a complicated process that inflates the creation and starting the production of, new engines. Computer simulation is widely used nowadays to solve this problem [1-3]. Such simulation requires finite-element models of the areas to be computed; building such models takes 40 to 80 percent of the total computation time $[4,5]$. This is due to the fact that when designing a new combustion chamber, one has to analyze a lot of various option before the most optimal one is chosen. For each option, they first have to build a geometric 3D combustion chamber model (a CAD model), then use it to build a finite-element (mesh) model. Creating the mesh model is additionally complicated due to the need to account for the combustion process aside from the geometry, as the computation mesh has to be further refined where the computable parameters are expected to change vastly. Therefore, we need a way to quickly edit the CAD model and rebuild the mesh model.

\footnotetext{
*Corresponding author: tr05@bk.ru
} 


\section{Materials and methods}

The paper describes the process of creating a parametric model of a 3D MGTE combustion chamber assembly and the internal volume of the computed sector. Parametric models can have their geometry adjusted quickly and without human intervention [6]. This advantage is made use of in various branches of mechanical engineering. Parametrizing the machine tooling used by multi-axial $\mathrm{CNC}$ machining centers makes designing such tooling much less time-consuming [7]. Another paper [8] cites the optimal design of a high-speed diesel combustion chamber on the basis of parameter space studies.

The authors hereof chose the popular Siemens NX package as their development environment. This CAD system is popular with aircraft-engine manufacturers. Creating a parametric assembly can be complemented with various methods [9], each having its pros and cons.

The modeling process programming method [6] consists in pre-compiling the geometric construction command lists in the form of a program code. When running the code in the NX interface, auxiliary sketches and part volumes are built automatically. Siemens NX supports various programming languages from its proprietary languages to a few common ones like UG/Open GRIP, SNAP, VB, C+, C\#, etc. Dimensions, geometric constraints, number of holes, and other source modeling data can be specified in a separate text file. By changing the parameters in the data file and running the program, we can produce a modified CC CAD model. The drawback of this method is the complexity of coding, especially when it comes to such complex-design assemblies as a CC. Programming a highly-branched algorithm to describe how an altered part would affect the size and positioning of other assembly components is also no simple task.

The traditional variational (dimensional) parametrization method is based on creating sketches with different parametric links superimposed [9]. The NX environment can create the so-called active dimensions that control the geometry. When specifying such a dimension, one creates a linked variable-value parameter. Changing an active dimension alters the distance or the angle between the components of the sketch, on which the dimension is based. Apart from dimensions, unambiguous geometry determination in NX requires such constratins as perpendicularity, coaxiality, tangency contraints, etc. The drawback of this method is that the model geometry has to be adjusted manually. The connecting dimensions of the parts in an assembly are not linked and have to be edited individually.

A better-developed variational parametrization method is referred to as the top-down modeling method [9, 10]. All the parts are created and edited in the context of their assembly. The connecting dimensions of components are inter-linked and are altered harmoniously. The top-down method, just like the programming method [6], supports the use of external data files while being less difficult to implement. It was this method that the authors hereof chose for their parametric CC 3D assembly effort.

\section{Creating the parametric 3D assembly of an MGTE combustion chamber}

The designed combustion chamber is intended for small-size gas-turbine aircraft engines. The small size actually complicates organizing the combustion process, which is why designing and finishing it requires thorough analysis of multiple options. The designed chamber is a ramjet ring chamber, see Fig. 1. Flame is stabilized in the toroidal recirculation zone. 


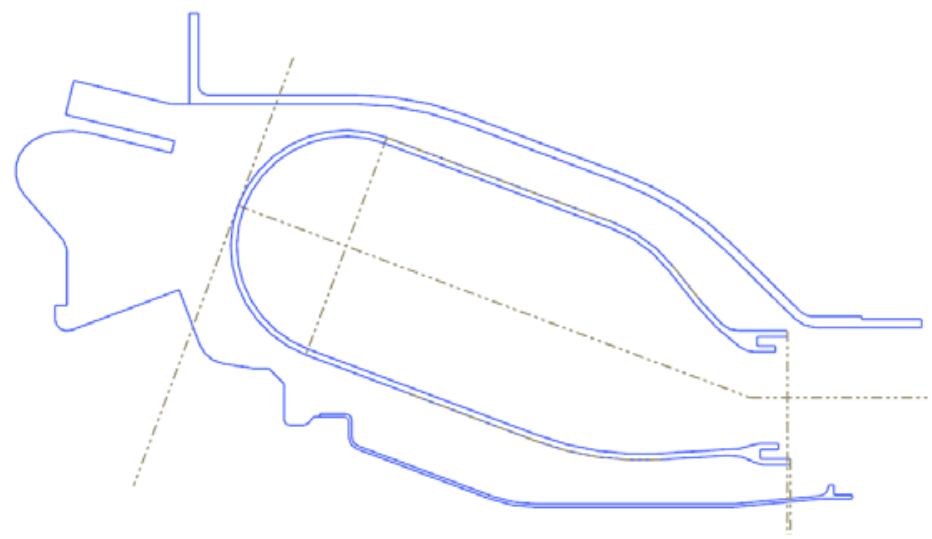

Fig. 1. Combustion chamber sketch

We first create an NX assembly file containing the control structure of the combustion chamber, consisting of three parts: the original structure, the working structure, and the resulting structure. The top-down method implies working with each structure consequentially.

The original structure must contain the assembly frame sketch. First we build the outerwall profile of the flame tube. It contains the geometric constraints on the sketch components, such as tangency, parallelism, collinearity, etc. Then the $\mathrm{CC}$ flame tube wall thickness is specified by means of equidistant profile displacement. Then we build the remaining elements of the combustion chamber.

While optimizing the $\mathrm{CC}$ design, we have to alter many dimensions of the assembly multiple times. Doing it in Siemens NX 3D modeling environment by finding the exact element we need is not the most convenient option. This is why we've created an external excel spreadsheet containing all the geometric parameters one might have to adjust. In the Expressions window of the NX interface, the dimensions of the model are linked to specific spreadsheet cells, see Fig. 2. This enables dimensional adjustments in Excel.

\begin{tabular}{|c|c|c|c|c|c|c|c|}
\hline$\angle$ & \multicolumn{2}{|l|}{ A } & \multicolumn{2}{|r|}{ B } & $\mathrm{C}$ & D & \\
\hline 1 & \multicolumn{2}{|l|}{ Name of the size } & \multicolumn{2}{|c|}{$\begin{array}{c}\text { Size } \\
\text { designation }\end{array}$} & Value & Dimension & \\
\hline 2 & \multicolumn{2}{|l|}{ Initial diameter } & \multicolumn{2}{|c|}{ Dn } & 314.00 & MM & \\
\hline 3 & \multicolumn{2}{|l|}{ Angle of the axis of the flame tube } & \multicolumn{2}{|c|}{ Teta1 } & 20.50 & $\circ$ & \\
\hline 4 & \multicolumn{2}{|l|}{ Width of the flame tube } & \multicolumn{2}{|c|}{ Hzt } & 76.00 & MM & \\
\hline 5 & \multicolumn{7}{|c|}{ Upper angle of the flame tube $n$} \\
\hline 6 & \multirow{2}{*}{\multicolumn{6}{|c|}{\begin{tabular}{r|l} 
Lower angle of the flame tube $n$ \\
Upper transition radius
\end{tabular}$\quad$ I Name }} & \multirow{2}{*}{ Value } \\
\hline 7 & & & & & & & \\
\hline 8 & Transition radius is lowe & 27 & Beta6 & \multicolumn{3}{|c|}{ ug_excel_read("C: |temp|Otv_Zhar_Trub.xisx","c39") } & -30 \\
\hline 9 & Distance to the upper transitiol & 28 & Beta7 & \multicolumn{3}{|c|}{ ug_excel_read("C: |temp|Otv_Zhar_Trub.xisx","C46") } & -45 \\
\hline 10 & Distance to the lower transitior & 29 & D1 & \multicolumn{3}{|c|}{ ug_excel_read("C: temp/Geom_zhar_Trub.xdsx","C11") } & 218 \\
\hline 11 & Diameter of the upper lor & 30 & D2 & \multicolumn{3}{|c|}{ ug_excel_read("C: Itemp|Geom_Zhar_Trub.xisx", "C12") } & 158 \\
\hline 12 & Diameter of the lower & 31 & Dd1 & \multicolumn{3}{|c|}{ ug_excel_read("C: Itemplotv_Zhar_Trub.xisx","C7") } & 0.8 \\
\hline 12 & Diameter of the lower loc & 32 & Dd2 & \multicolumn{3}{|c|}{ ug_excel_read("C:|temp|Otv_Zhar_Trub.xiss"," "C14") } & 0.8 \\
\hline 13 & Distance to the upper cast & 33 & Dd3 & \multicolumn{3}{|c|}{ ug_excel_read("C: |temp|Otv_Zhar_Trub.xlsx","C21") } & 0.8 \\
\hline 14 & Distance to the lower cast & , 34 & Dd4 & \multicolumn{3}{|c|}{ ug_excel_read("C: |temp|Otv_Zhar_Trub.xisx","C28") } & 0.8 \\
\hline 15 & Thickness of the flame tul & 35 & Dd5 & \multicolumn{3}{|c|}{ ug_excel_read("C: |temp|Otv_Zhar_Trub.xisx","C35") } & 0.8 \\
\hline & & 36 & Dd6 & \multicolumn{3}{|c|}{ ug_excel_read("C: |temp|Otv_Zhar_Trub.xisx","C42") } & 0.8 \\
\hline
\end{tabular}

Fig. 2. Siemens NX to Excel link 
The sketches created as a part of the original structure are then copied to the working structure be mans of the WAVE Geometry Linker. They are therefore used to create threedimensional solids of rotation. A few holes are made in the flame tube. The axes of the holes in the front wall are located at an angle to the normal of the outer tube surface, see Fig. 3. The angles for each hole row as well as other parameters are read from the linked excel spreadsheet.

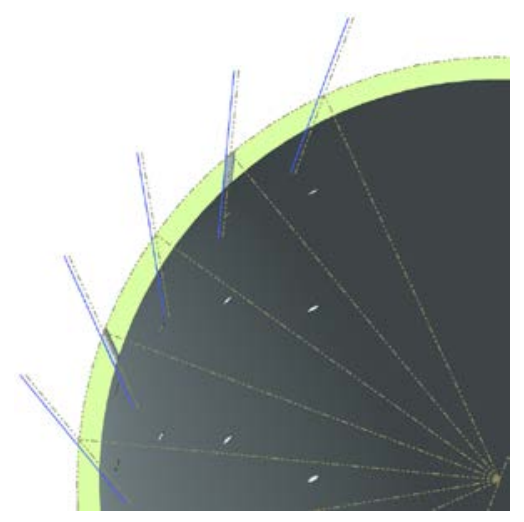

Fig. 3. Holes in the front wall of the flame tube

Finally, all the components of the assembly are copied to the resulting structure where they are saved as individual files. Figure 4 presents the finished parametric model of the 3D CAD assembly of an MGTE combustion chamber. It complrises the external housing outlines, the flame tube structure (with cooling and air supply holes), and the diffuser output.

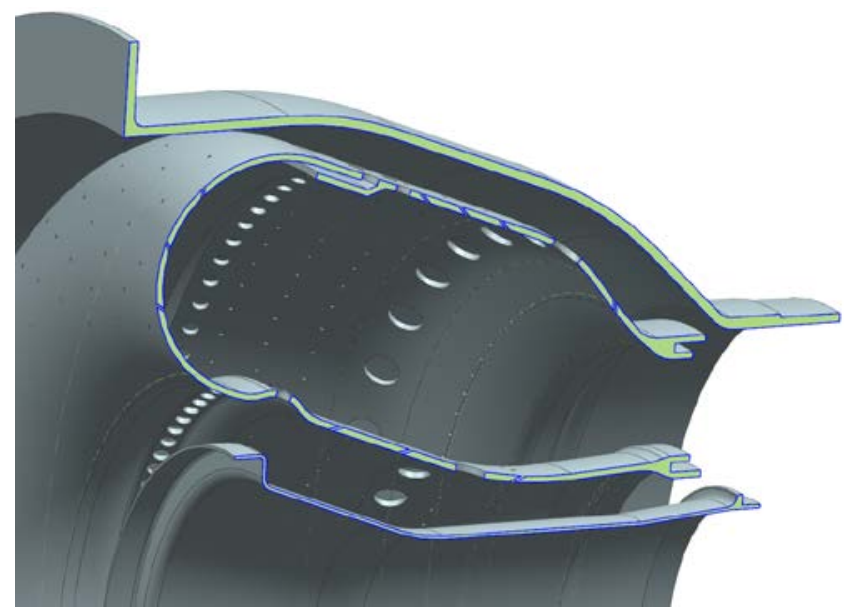

Fig. 4. Parametric 3D CAD assembly of a combustion chamber: final version

When modeling the CC assembly, we've taken into account a number of constraints that the combustion process imposes on the design. We've found the values of, and sticked to, the limits on distances between cooling/mixing/air supply holes, closures, hole diameters, and inter-hole pitches. We have also stayed within the limits on the combustion chamber length, middel section height, inclination angle, flame tube wall thickness, etc. 


\section{Deriving the internal volume of the computed sector}

For numerical analysis of inter-chamber processes, one uses 3D models of CC internval volumes and channels. In our case, the problem is axis-symmetric, and it makes sense to analyze just a single-burner sector rather than whole chamber to save computation time.

The desired model is based on a parametric assembly and the profiles created as a part of the original structure, see Fig. 1. First, the volume is created by means of the chamber outline. Boolean subtract operation is used to remove the solids of flame tube walls and other CC components. The resulting geometry is reduced to the size of a single-burner sector, see Fig. 5.

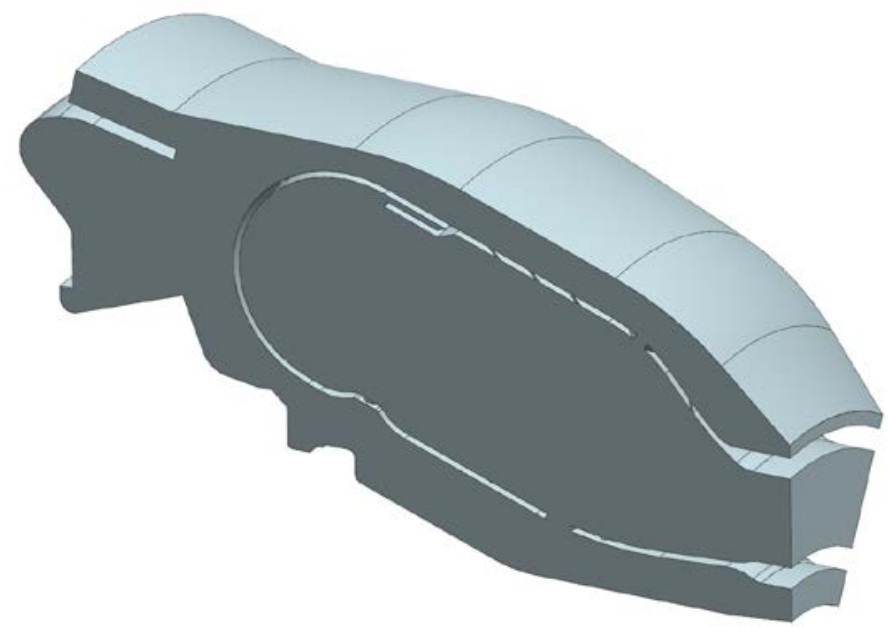

Fig. 5. Internval volume of the computed combusion chamber sector

We've used the proposed method to build several combustion chamber designs, then refined them. Compared to traditional remodeling, this method is about $50 \%$ less timeconsuming.

\section{Conclusion}

The developed combustion chamber assembly parametrization and computer section building has been tested and found:

1. To considerably simplify the simultaneous building of multiple $3 \mathrm{D}$ combustion chamber models differing insignificantly in size.

2. To enable model editing without running the NX interface thanks to an external parametric excel spreadsheet. The file can be auto-edited by the optimizer software [11] without human intervention.

3. Changes in the assembly do affect the linked computed sector of the CC. In theory, we can build a system that rebuilds the mesh model.

4. The created parametric 3D assembly of an MGTE combusion chamber can be used for the geometric optimization of combustion chambers (housings and flame tubes) by means of special software like IOSO [11].

5. The developed method halved the time consumption of computation and refinement. 
This work was supported by the Ministry of education and science of the Russian Federation in the framework of the implementation of the Program "Research and development on priority directions of scientific-technological complex of Russia for 2014-2020" (RFMEFI58716X0033).

\section{References}

1. I.V. Kurchatkin, A.A. Gorshkalev, E.V. Blagin, Methods of the working processes modelling of an internal combustion engine by an ANSYS IC Engine module, Journal of Physics: Conference Series, v. 803(1), (2017)

2. Y. Levy, A. Gany, Y. Goldman, V. Erenburg, V. Sherbaum, V. Ovcharenko, L. Rosentsvit, B. Chudnovsky, A. Herszage, A. Talanker, Increasing operational stability in low NOx GT combustor by a pilot flame, Proceedings of the ASME Turbo Expo, v. 2(A and B), pp. 645-654 (2010)

3. P.-Q. Guo, S.-S. Zang, B. Ge, Large eddy simulation of reactive flow in a bluff-body combustion chamber, Kung Cheng Je Wu Li Hsueh Pao/Journal of Engineering Thermophysics, v. 31(11), pp. 1969-1972 (2010)

4. V.M. Anisimov, I.A. Zubrilin, M.Y. Orlov, Investigation of thermal and stress states of the annular combustion chamber flame tube walls. Proceedings of the ASME Turbo Expo, v. 4B, (2016)

5. M.Y. Orlov, I.A. Zubrilin, R.A. Zubrilin, The Calculated Estimate of Pressure Oscillations behind the Power Plant Combustion Chamber Burner, Procedia Engineering, v. 176, pp. 394-401 (2017)

6. A.I. Ryazanov, Automated $3 D$ modeling of working turbine blades, Russian Engineering Research, v. 36 (9), pp. 751-754 (2016)

7. A.V. Balaykin, K.A. Bezsonov, M.V. Nekhoroshev, A.P. Shulepov, Developing Parametric Models for the Assembly of Machine Fixtures for Virtual Multiaxial CNC Machining Centers, IOP Conference Series: Materials Science and Engineering, v. 302(conference 1), (2017)

8. A.N. Vrublyovsky, Ye.S. Graivoronsky, Optimal design of a diesel combustion chamber in a multicriterial problem statement (Rezultaty optimalnogo proyektirovaniya kamery sgoraniya dizelya $v$ mnogokriterialnoy postanovke zadachi), Road Transport (Avtomobilny transport), v. 37, pp. 7-12 (2015)

9. A.I. Ryazanov, Ye.S. Goryachkin, Solid parametric CAD modeling in Siemens NX (Tverdotelnoye parametricheskoye CAD modelirovaniye $v$ Siemens NX), Electronic textbook, Samara: SSAU, p. 148 (2014)

10. Z. Pan, X. Wang, R. Teng, X. Cao, Computer-aided design-while-engineering technology in top-down modeling of mechanical product, Computers in Industry, v. $\mathbf{7 5}$ pp. 151-161 (2016)

11. I.N. Egorov, G.V. Kretinin, I.A. Leshchenko, Robust design optimization strategy of IOSO technology, Fifth World Congress on Computational Mechanics, pp. 1-8 (2002) 